\title{
Quantum Imaging X-ray CT Systems Based on GaAs Radiation Detectors Using Perspective Imaging Reconstruction Techniques
}

\author{
J. Přibil ${ }^{1}$, B. Zat'ko ${ }^{2}$, I. Frollo ${ }^{1}$, F. Dubecký ${ }^{2}$, P. Ščepko ${ }^{3}$ and J. Mudron̆ ${ }^{4}$ \\ ${ }^{1}$ Institute of Measurement Science, SAS, Dúbravská cesta 9, SK-841 04 Bratislava, Slovakia, umerprib@savba.sk \\ ${ }^{2}$ Institute of Electrical Engineering, SAS, Dúbravská cesta 9, SK-841 04 Bratislava, Slovakia \\ ${ }^{3}$ T\&N System, Ltd., Severná 5, SK-974 01 Banská Bystrica, Slovakia \\ ${ }^{4}$ MTC, a. s., Kuzmányho 11, SK-031 01, Liptovský Mikuláš, Slovakia
}

\begin{abstract}
The work presents two generations of developed portable quantum $\mathrm{X}-\mathrm{CT}$ mini-systems which utilize monolithic semi-insulating GaAs detectors. This contribution describes the present status of the assembling of the new portable X-ray CT mini system. Developed modification of the $\mathrm{X}$-ray image reconstruction based on perspective imaging techniques has been experimentally verified on testing phantoms and practically implemented for processing images of real test objects. Performed measurement of the performance of the SI GaAs detectors and the integral spectra of ASIC DX64 readout chips are also mentioned.
\end{abstract}

Keywords: $\mathrm{X}$-ray imaging, single photon counting detector, image reconstruction

\section{INTRODUCTION}

$\mathrm{T}$ HE X-ray computer tomography $(\mathrm{X}-\mathrm{CT})$ is a nondestructive testing method able to evaluate the inner structure of investigated objects [1], [2]. The crosssectional imaging is achieved by mathematical reconstruction of projections of a tested object. Such a projection is an intensity image of transmitted X-ray photons through the evaluated object. Small CT imaging instruments for the evaluation of small animals have been developed preferably for operation in the positron emission mode (PET). Almost all commercial X-CT systems use photodiodes as detectors that are covered by a scintillator [3].

This paper makes a comparison of two developed portable quantum X-CT mini-systems (XCTMS) which utilize monolithic semi-insulating (SI) GaAs detectors. The solution of these two X-CT devices was also different in the approach of sample and X-ray source/detector arrangement. Both devices are using the same principle of image reconstruction from projections [4], but modifications of the basic reconstruction algorithm to eliminate some imperfections resulting from physical properties of used X-ray detector and utilized scanning technique must be applied [5].

\section{SUBJECT \& METHODS}

The first generation of realized $\mathrm{X}-\mathrm{CT}$ device used the construction solution, where the sample is rotated and can be linearly moved on the $x$-axis for changing the reconstruction geometry (zoom in/zoom out), the $\mathrm{X}$-ray source and detector are stationary [5]. This experimental equipment consists of:

- active X-radiation source with energy of photons up to 80 $\mathrm{keV}$ based on X-ray glass tube Oxford P/N XTG 90503 Model SB-80-500 SourceBlock, (integrated tube housing assembly) [6],

- digital X-ray scanner consisting of 17 monolithic 24 strip line detectors named "SAMO" based on bulk SI GaAs [7],

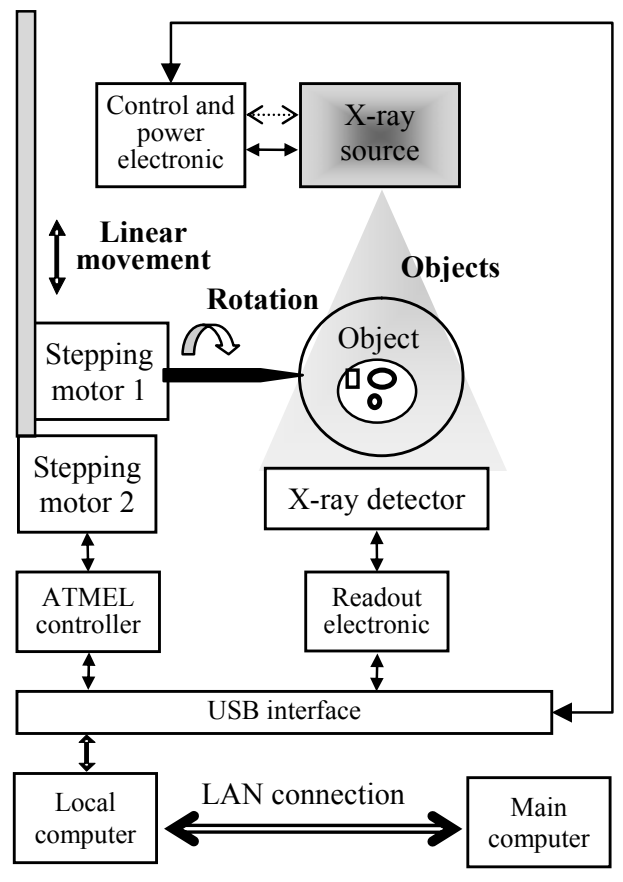

Fig. 1 Block diagram of the $1^{\text {st }} \mathrm{X}-\mathrm{CT}$ device

- measured sample positional system (for movement on $x$ axis and rotation),

- CCD camera with infra LED lighting for condition monitoring inside of a scanning area,

- local (remote) PC computer for parallel control of all working devices (X-ray source, scanner, positional system and CCD camera) connected via serial or USB lines (through USB-to-Serial converters),

- main PC located in another place (office room) for distributed control of performed X-ray experiments connected via LAN network to the local PC (see Fig. 1).

The whole X-ray scanning area was incased to a box shielded by $1.5 \mathrm{~mm} \mathrm{~Pb}$ plate. Achieved position resolution of our first testing X-CT mini platform was up to $0.1 \mathrm{~mm}$ on $x$-axis, 
minimum rotation angle was $1.8 \mathrm{deg}$, and the investigated object can rotate in full interval of $0 \div 360 \mathrm{deg}$ [8] withal.

In the second generation of XCTMS the different construction approach was applied. An evaluated object is located between the X-ray source and the detection unit. One of the stepper motors ensures a full rotation of the $\mathrm{X}$-ray source $\&$ detection unit coupled system around the object (see Fig. 2a). The other stepper allows for its linear positioning along the $z$ direction. As a fan-beam configuration (diverging beam) is used, the micro-adjustable slit ensures that the width of the X-ray beam is little wider than the detector double array width and irradiates only the active area of the pixel detectors.

a)

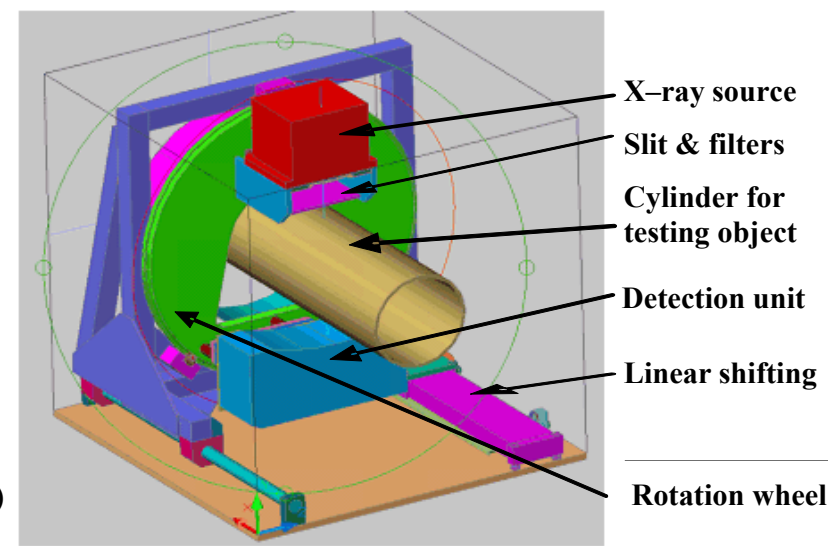

b)

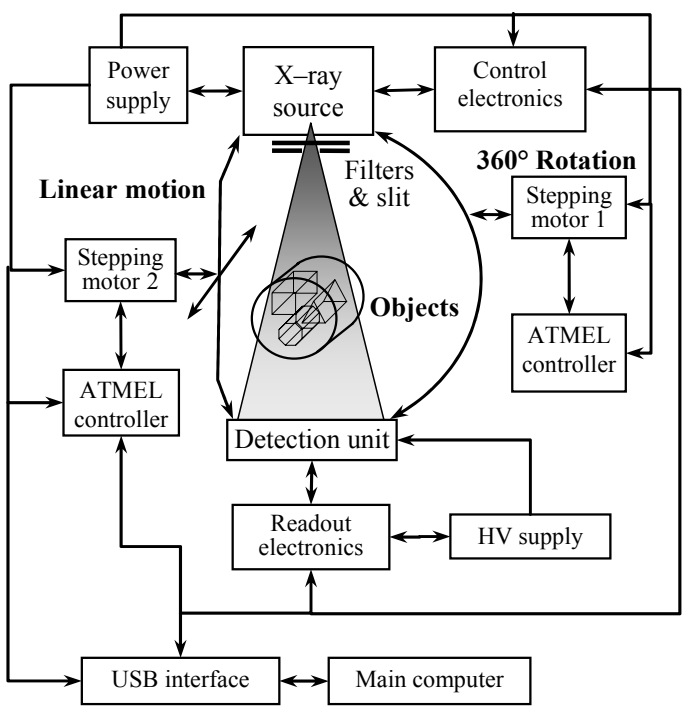

Fig. 2 The developed new portable XCTMS: 3-D visualization (a), control block diagram (b).

The mechanical construction of the XCTMS has the following structure: the X-ray source, connected with the filter and the slit holder, is positioned in the top part of an aluminium wheel, which is driven by the stepper motor. The cylindrical holder is used to fix the objects tested. This XCTMS device is portable and consists of the following parts (see block diagram in Fig. $2 \mathrm{~b}$ ): the X-ray source, the detection unit, two stepper motors, which control the rotation and the linear movement of the $\mathrm{X}$-ray source coupled with the detection unit around the object evaluated, and a local high-performance PC with a touch-screen monitor allowing for comfortable control of all system elements.

The X-ray source (Source-Ray Inc.: Model SB-80-500) with a tungsten anode operates in an accelerating voltage range of 35$80 \mathrm{kV}$ at a maximum current of $500 \mu \mathrm{A}$ with a maximum focal

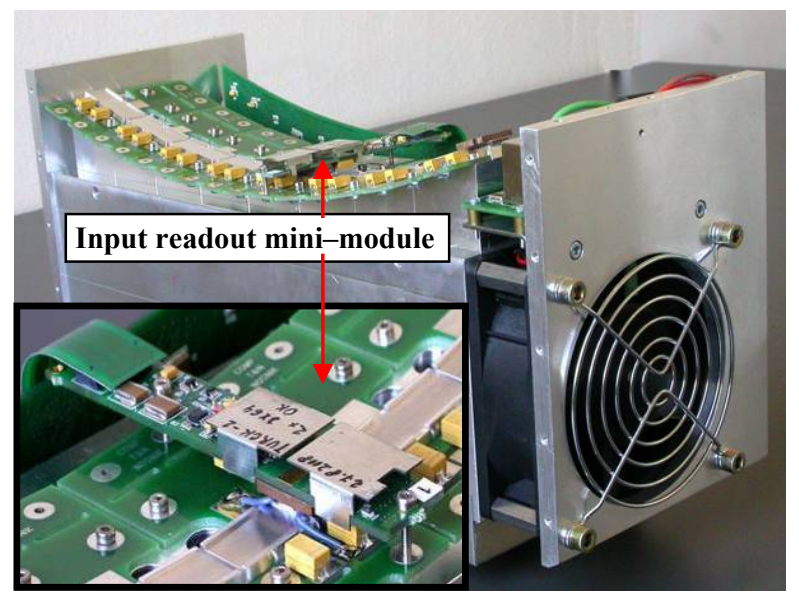

Fig. 3 Photo of the detection unit with the input readout minimodule (inset).

spot size smaller than $46 \mu \mathrm{m}$ at a maximum power of $40 \mathrm{~W}$ [6]. $\mathrm{X}$-rays generated by the source pass through two filters, selectable from $2 \times 8$ positions in two rotatable carousels, followed by a micro-adjustable slit, which limits the width of the cone (into which $\mathrm{X}$-ray photons are emitted) into a narrow, almost one-dimensional beam. The detection unit consists of 16 input mini-modules, each with $2 \times 64$ monolithic SI GaAs pixel detectors with a pitch of $250 \mu \mathrm{m}$ arranged along two lines on the chip and assembled into an arc (see Fig. 3). The detection double array incorporates $2 \times 1024$ pixels over a total length of $261.5 \mathrm{~mm}$. A small gap, corresponding to 1.5 pixels, is maintained between each two neighbouring modules to prevent the outer pixels from damage. The total length of the double array on the chip is $16.25 \mathrm{~mm}$. The operational bias of the SI GaAs detectors is ranged between 150 and $300 \mathrm{~V}$. The pixel detectors are dc-coupled to the inputs of two ASICs-type DX64 readout chips [8], by wire bonding via pitch adapters, which adjust the pitch of the pixel detectors to the input pads of the ASICs. The readout chips have two discrimination levels and 20-bit counters for each channel; hence one energy window is available within one evaluation scan. The devices are glued and wire-bonded onto an input PCB (printed circuit board) with dimensions of about $16 \mathrm{~mm} \times 120 \mathrm{~mm}$. The PCBs are fixed onto Peltier coolers, which stabilize the working temperature of the detectors and the input electronic circuitry. 


\section{IMAGE RECONSTRUCTION FROM PROJECTIONS}

There are many different methods used for image reconstruction from projections - filtered backprojection (FBP), reconstruction via frequency domain or many kinds of iterative algorithms. We have used FBP because of sufficient speed to accuracy ratio [1]. Considering setup geometry, three different arrangements are possible: parallel beam (see Fig. 4a), fan beam with equidistal detectors (Fig. 4b) and fan beam with equiangular detectors (Fig. 4c).
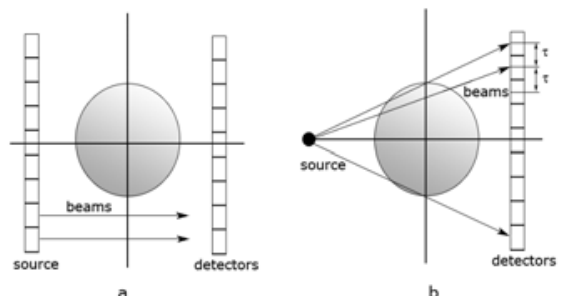

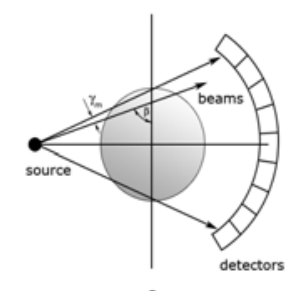

c
Fig. 4 Basic arrangements for different types of projection measurement.

The output of transformation from spatial domain to Radon domain is called sinogram $-\mathrm{p}(\tau, \theta)$

$$
p(\tau, \theta)=\int_{-\infty}^{\infty} \int_{-\infty}^{\infty} f(x, y) \delta(x \cos \theta+y \sin \theta-\tau) d x d y,
$$

where $\theta$ is the angle of particular projection, $\tau$ is the target distance of each beam and $f(x, y)$ is a $2 \mathrm{D}$ function to be projected. Filtered backprojection can be expressed by the following equation

$$
f(x, y)=\int_{0}^{\pi} q(x \cos \theta+y \sin \theta, \theta) d \theta,
$$

where $q$ is a 2D function of filtered projections. Filters implemented in our algorithms were Ram-Lak, Shepp-Logan, Hann, Hamming and cosine filter. The fan-type projections must be rebinned and interpolated to parallel-type before applying back projection algorithm. It follows from the figure geometry that for any ray $\tau=R \sin \alpha$ and consequently

$$
r_{\beta}(\alpha)=p_{(\alpha+\beta)}(R \sin \alpha) .
$$

Nevertheless filtered back projection needs many projections (several hundreds or more) to obtain a correct image because of many effects coming into $\mathrm{X}$-ray sinogram such as fluorescence, $\mathrm{X}$-ray scattering, beam hardening, phase effects, etc. Perspective approach uses an iterative scheme of tomographic reconstruction. Its advantage is that we can get a good image even from noisy data and a low number of projections [1], [4].

In the first generation of the $\mathrm{X}-\mathrm{CT}$ device, the parallel beam or fan beam with equidistal detectors geometry can be used [9], (see demonstration example with testing phantom in
Fig 5). For the new XCTMS with rotating X-ray source and detection unit system, the fan beam with equiangular detectors geometry will be applied.

a)

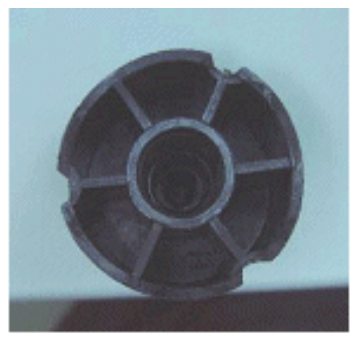

b)

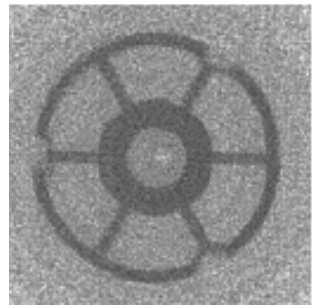

c)

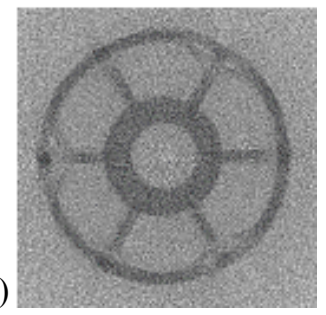

Fig. 5 Image reconstruction of the testing object: photography of a plastic tube with six capsules, diameter $40 \mathrm{~mm}$ (a), finally reconstructed image by the parallel beam method -100 projections per $3.6 \mathrm{deg}(\mathrm{b})$, reconstructed image by fan beam method (c).

\section{ModificAtion of Image ReCONSTRUCtion Algorithm}

To provide good $\mathrm{X}$-ray images some imperfections must be eliminated. The imperfections follow from physical properties of used devices and the applied scanning technique. The basic scanning and image reconstruction process used in our experiments can be divided to four steps [5]:

1. Finding the optimal settings of X-ray source and scanner operating parameters.

2. Performing the scans of a tested sample with set number of projections (given by selected rotation step angle and chosen $x$ - axis slide position).

3. Collecting data of performed scans including the preprocessing operations.

4. Image reconstruction from obtained object projections.

Prior to the experiment, the first scan without the tested object must be performed. This scan contains information about actual background (in virtue of sensitivity of the X-ray detector) and these data are applied for building of the correction matrix. The correction matrix is subsequently applied to all next scans with the tested object.

To observe a possible inaccuracy of the sample position, the testing phantom can be used. In our case, the placement of inserted metal pins in the testing phantom (one in the middle, second by the border of plastic tube with the maximum diameter) was chosen. These features can be covered by indirect method, for example by the sinogram of the tested object calculated as (1). When the sample position and rotation is correct, the sinogram obtains a direct line located at the medium (derived by inserted middle pin) and pure sinus 
curve (derived by the border pin). From the sinogram in Fig. 6 it is evident, that this condition is not fulfilled. Therefore, some correction curve for linearization and rotation of the data matrix must be applied.
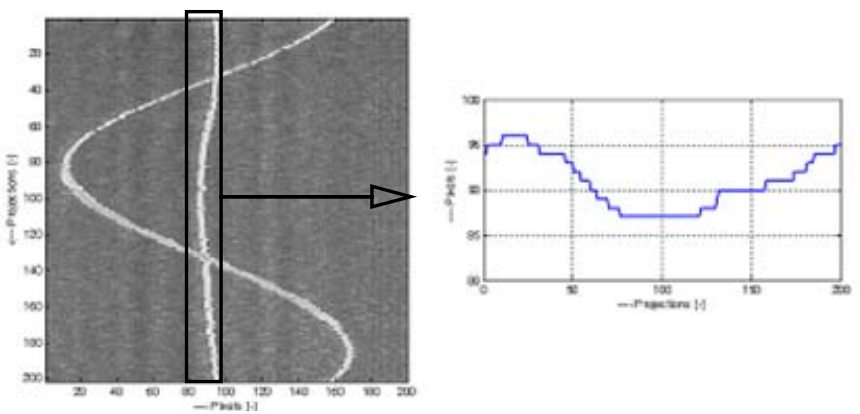

Fig. 6 Sinogram of testing phantom of 200 projections per $1.8 \mathrm{deg}$ angle with dimension of 200 pixels (left), derived correction curve (right).

The used type of SI GaAs detector generates the frequency noise, which also depends on the temperature. The high frequency component of this noise brings a "background" texture of the reconstructed object. For that reason, the lowpas (LP) filtration on the slices of a projection data matrix can be performed. On the other hand, the filtration produces the blur edge effect. Therefore, the parameters of the designed low-pas filter must be set as a compromise between the two mentioned antagonistic requirements (see Fig. 7).

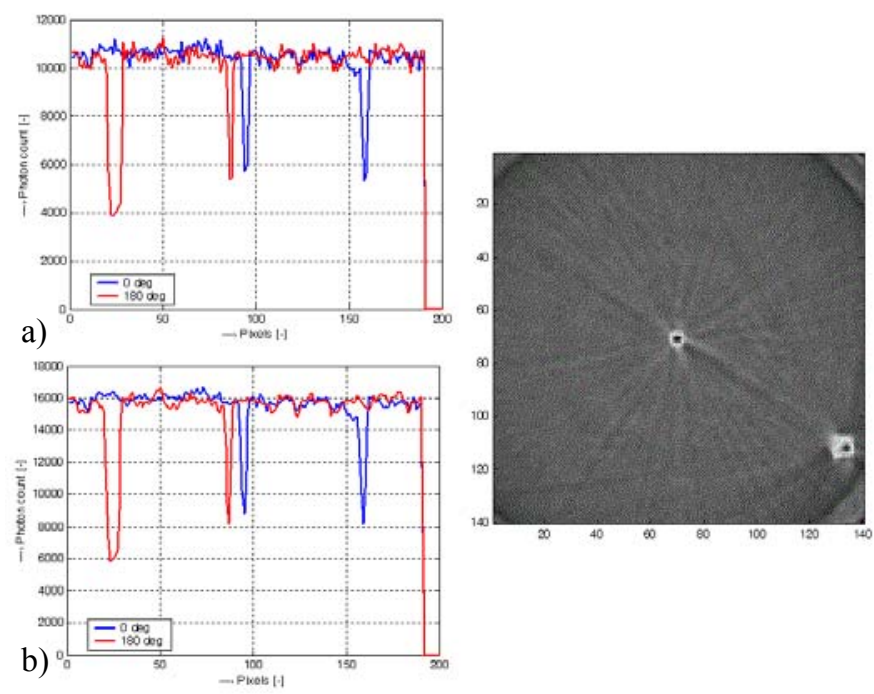

Fig. 7 Demonstration of LP filtration $-H_{L P}(z)=1 /\left(1+0.89 z^{-1}\right)$ : original projections of 0 and $180 \mathrm{deg}$ angle on the cut position of 289

(a), projections after application of the LP filter (b), resulting reconstructed image with LP filtering (right).

Algorithms for image reconstruction were written in $\mathrm{C}++$ language as a console application. Parameters that could be passed to the algorithm are related to the geometry used (e.g. inter-detector distance, source-to-detector distance, source-to- object-center, angle of fan, number of projections and samples). To get the projection data, 200 projections and depth of 200 samples were used. Source-to-detector distance was $600 \mathrm{~mm}$; source-to-object-center was $100 \mathrm{~mm}$.

\section{Measurement of Si GaAs Detector Performance}

The main characteristics of the ASIC DX64 readout chip are published in [8]. Total number of channels is 64. Each channel consists of the charge sensitive preamplifier, shaping amplifier and two discriminators with two independent 20 bits counters. Power dissipation per channel is $5 \mathrm{~mW}$. Peaking time of the shaped impulse is $160 \mathrm{~ns}$ and the equivalent noise charge for $\mathrm{C}_{\mathrm{det}}=1 \mathrm{pF}$ is $110 \mathrm{e}^{-} \mathrm{rms}$. The maximum counting rate of the readout chip is more than $1 \mathrm{MHz}$ per channel. Used detectors have Schottky contacts with four different diameters $(0.75,0.50,0.30$ and $0.20 \mathrm{~mm})$. On the other side of the wafer the AuGeNi eutectic alloy with a thickness of $100 \mathrm{~nm}$ was evaporated. In our experiments we used eight detectors with four different diameters of the Schottky contact.

The measured reverse $\mathrm{I}-\mathrm{V}$ characteristics of all detectors at RT are depicted in Fig. 8. The breakdown voltage increases with decreasing of detector area while the reverse saturation current is reduced from $30 \mathrm{nA}(0.75 \mathrm{~mm}$ contact $)$ down to $5 \mathrm{nA}(0.20 \mathrm{~mm}$ contact $)$ at operating reverse bias voltage of $220 \mathrm{~V}$, which corresponds to the current density of about $6.8 \mu \mathrm{Acm}^{-2}$.

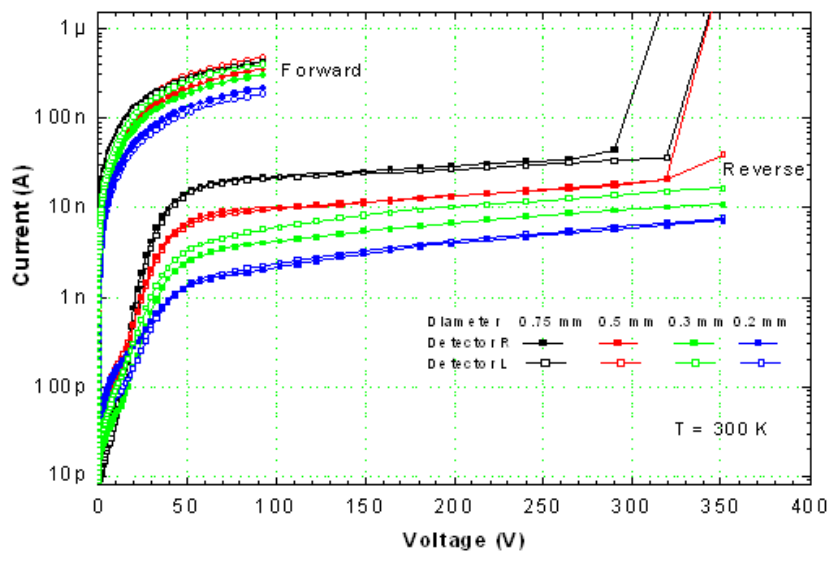

Fig. 8 Current-voltage characteristics of fabricated detectors.

Wire bonded detectors to the inputs of the readout chip are shown in Fig. 9. Only eight channels of the readout chip have been used in the present study. The reverse bias voltage of $220 \mathrm{~V}$ was applied to detectors and the radioisotope ${ }^{241} \mathrm{Am}$ was used as a $\gamma-(59.5 \mathrm{keV})$ and $\mathrm{X}$-rays $(13.9,17.8$ and $20.8 \mathrm{keV}$ ) source.

Measured integral spectra (dependence between the total count of the channels and adjusted threshold) of SI GaAs detectors are demonstrated in Fig. 10. Right edge of each spectrum represents impulses generated by $59.5 \mathrm{keV} \gamma-$ photons. 


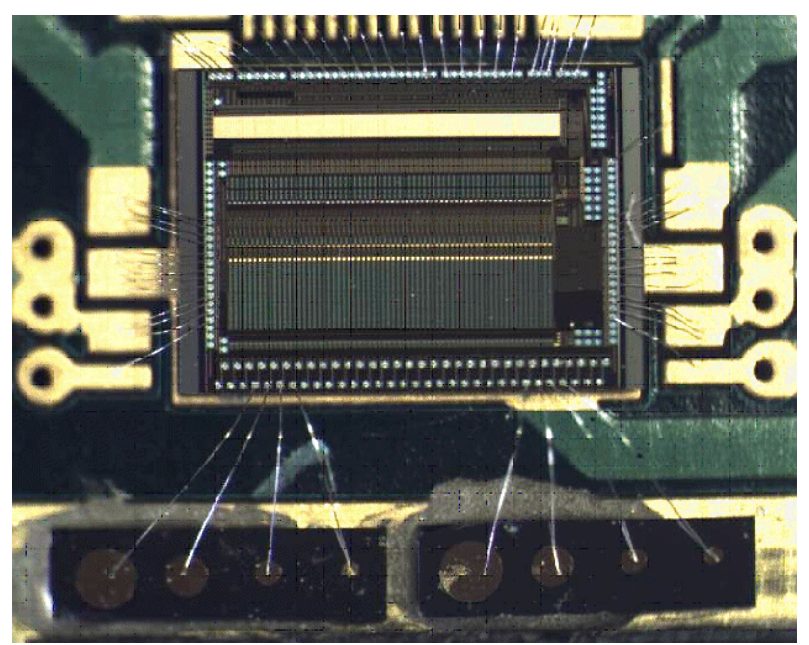

Fig. 9 Photo of SI GaAs radiation detectors of circular shape with different diameter coupled to ASIC DX64 readout chip.
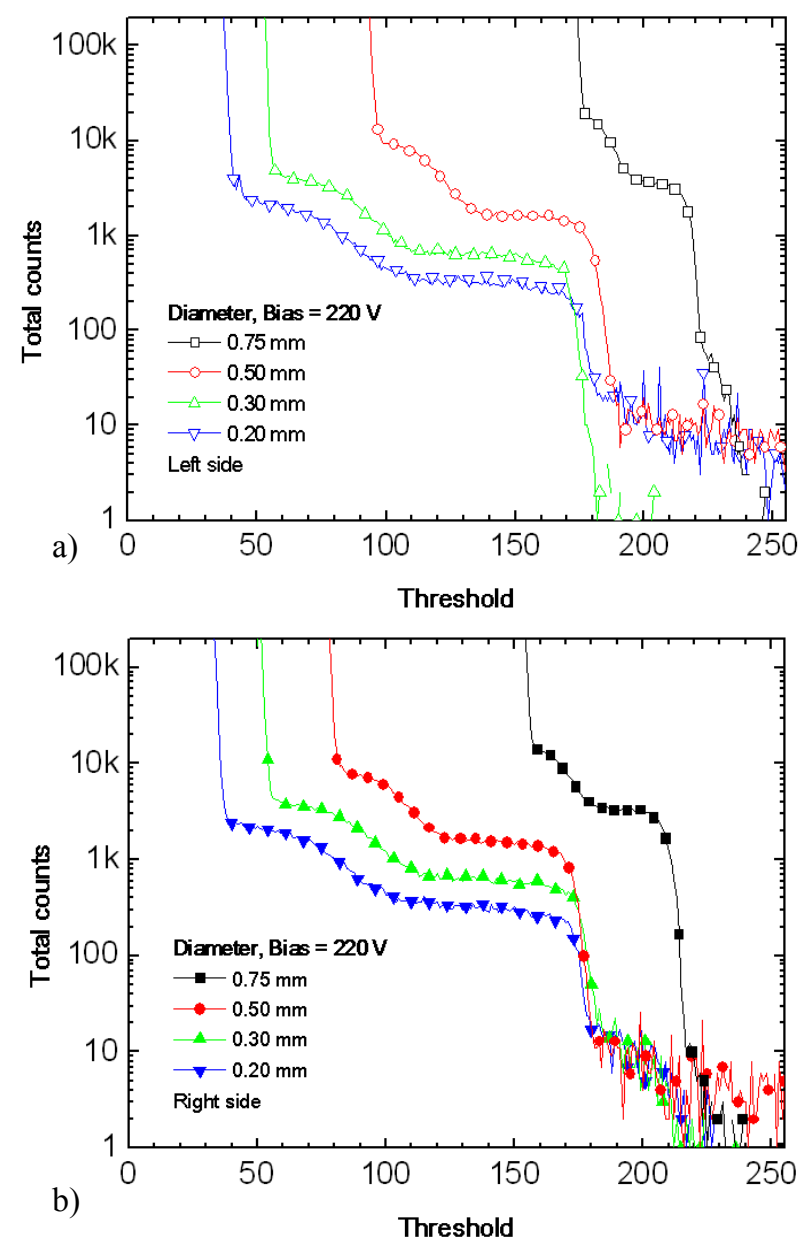

Fig. 10 Integral spectra of ${ }^{241} \mathrm{Am}$ : detector set 1 (a), and detector set 2 measured using ASIC DX64 readout chip (b).
Considering detectors DC-coupled to the ASIC DX64 readout chip, it is important to have the smallest possible reverse current because it is a source of noise and causes the shift of working point of the input preamplifier. The noise edge is rising with the detector area due to the increase of the reverse current. The dynamic range is almost the same for all channels with detectors but the gain is falling nearly three times and the offset is boosted with the rising of detector reverse current. The situation is becoming critical if the current exceeds $15 \mathrm{nA}$, because, in this case, the gain is about three times lower compared to the nominal value.

Fig. 11 shows calculated differential spectra of each tested channel. The energy resolution in FWHM (Full Width at Half Maximum) is better for the detector with the smaller Schottky contact. The best value of $3.4 \mathrm{keV}$ FWHM has been obtained with the smallest detector.
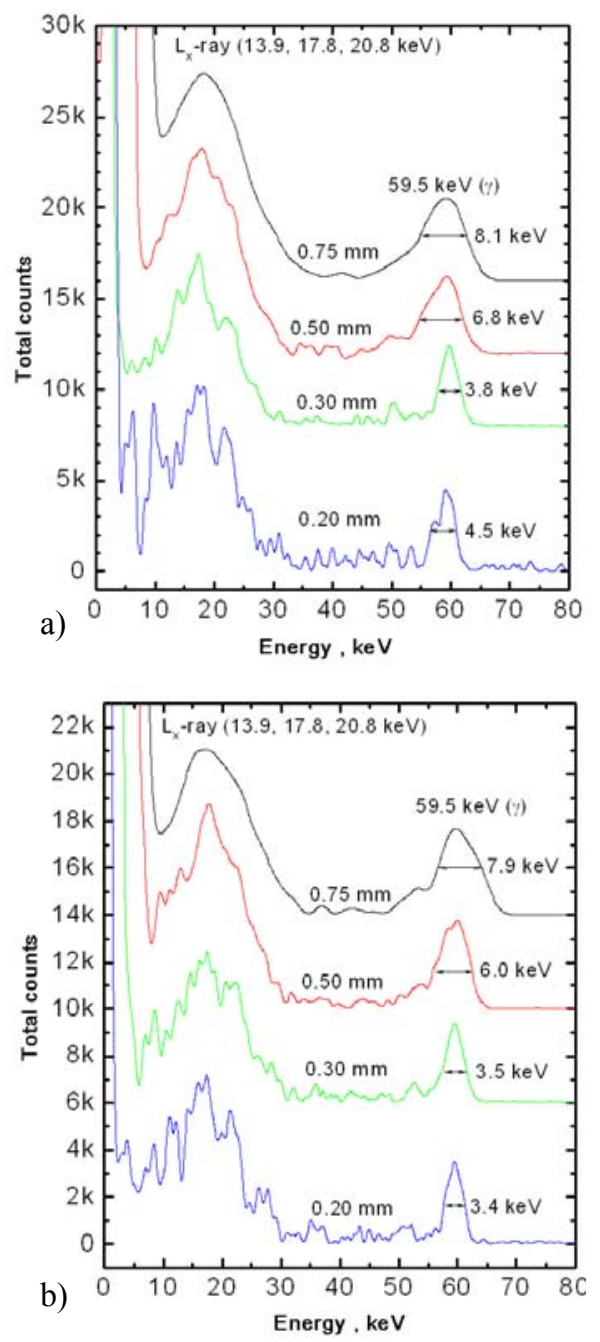

Fig. 11 Calculated differential spectra of ${ }^{241} \mathrm{Am}$ measured by two sets of SI GaAs detectors using ASIC DX64 readout chip: set 1 (a), and set 2 (b). Evaluated energy resolution is in FWHM of $59.5 \mathrm{keV}$ $\gamma$-photons. 
The obtained measurement results show that X-ray photons $(14 \div 21 \mathrm{keV})$ generated by ${ }^{241} \mathrm{Am}$ radioisotope source are resolved by detectors with contact diameter below $0.50 \mathrm{~mm}$ but due to the small area of the contact measured spectra show high level of statistical fluctuation in total counts.

\section{CONClusion}

Two portable quantum $\mathrm{X}-\mathrm{CT}$ mini-systems based on monolithic GaAs radiation imaging detectors have been realized. The current assembling system is able to operate as an $\mathrm{X}$-ray scanner or $\mathrm{X}-\mathrm{CT}$ equipment with fixed objects up to a diameter of $180 \mathrm{~mm}$ and $250 \mathrm{~mm}$ in length. The detection part consists of a double array of $2 \times 1024$ SI GaAs detectors with a pitch of $250 \mu \mathrm{m}$. Two discrimination levels of the readout chip enable the energy selection of incident photons. A fine tunable slit mounted behind the $\mathrm{X}$-ray source output guarantees a minimal dose for living objects. The expected spatial resolution of the device is $125 \mu \mathrm{m}$. The complete assembled $\mathrm{X}-\mathrm{CT}$ equipment on the electro-mechanical point of view is shown in Fig. 12.

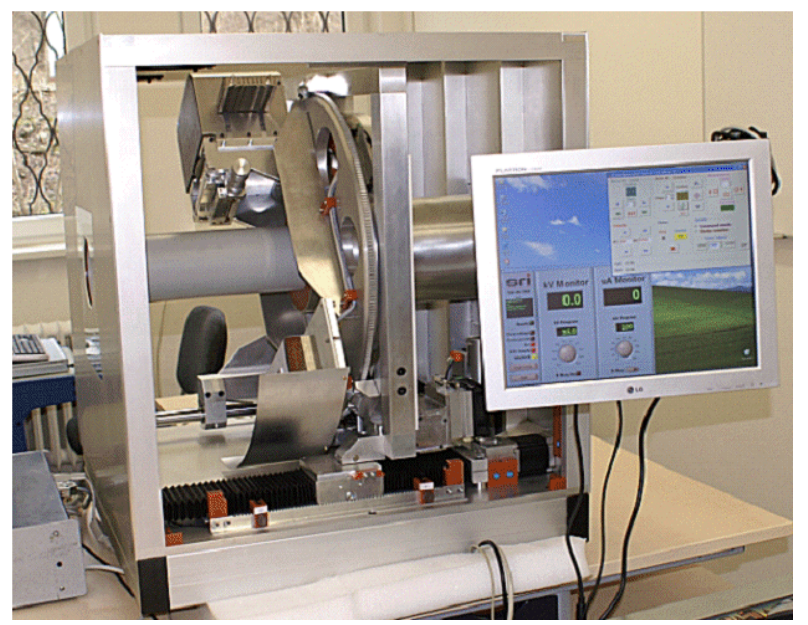

Fig. 12 Photo of developed $\mathrm{X}-\mathrm{CT}$ equipment including the main control computer with touch-screen LCD panel.

Developed modification of the $\mathrm{X}$-ray image reconstruction based on perspective imaging techniques has been experimentally verified on testing phantoms and practically implemented for processing images of real test objects. The basic reconstruction algorithm was supplemented with modifications to eliminate some imperfections following from physical properties of the used X-ray detector and applied scanning technique.

The measurement of the integral spectra of ASIC DX64 readout chips used in X-ray detector was performed. It was found out that detectors are heated by the readout chip to about $35{ }^{\circ} \mathrm{C}$ due to their gluing close to the chip on the same support. This caused significant current rising. To prevent overheating of detectors we need to increase the distance between detectors and the readout chip. Using the solid state
Peltier cooler could ensure a lower detector operation temperature, e.g. $15^{\circ} \mathrm{C}$ that results in substantial lowering of the detector current about one order of magnitude. Moreover, also the noise level and offset of detected spectra decreases and the gain of the readout channel increases to nominal value.

\section{ACKNOWLEDGMENT}

This work was partially supported by the Agency for Promotion of Research and Development under contracts no. APVV-0459-06 and APVV-99-P06305, and by the Grant Agency of the Slovak Academy of Sciences, project VEGA no. $2 / 0142 / 08$.

\section{REFERENCES}

[1] Kak, A.C., Slaney, M. (1999). Principles of Computerized Tomographic Imaging. New York: IEEE Press.

[2] Jakůbek, J. (2007). Data processing and image reconstruction methods for pixel detectors. Nuclear Instruments and Methods in Physics Research A, 576, 223-234.

[3] Wu, H. et al. (2008). In IEEE NSS-MIC 2008 Nuclear Science Symposium - Medical Imaging Conference. Book of Abstracts. IEEE, 545.

[4] Jan, J. (2006). Medical Image Processing, Reconstruction and Restoration: Concepts and Methods. Taylor \& Francis Group.

[5] Přibil, J. et al. (2008). Experiments with application of image reconstruction method based on perspective imaging techniques in X-ray $\mathrm{CT}$ mini system. In Proceedings of the $15^{\text {th }}$ International Conference IWSSIP '08. Bratislava: STU, 33-36.

[6] Source-Ray, Inc. (2002). The Model SB-80-500 SourceBlock Installation/Operation Manual.

[7] Dubecký, F. et al. (2006). First results observed with test X-CT system using GaAs radiation detector working in single photon counting regime. In Proceedings of the $6^{\text {th }}$ International Conference on Advanced Semiconductor Devices and Microsystems ASDAM '06. Bratislava: STU, 213-216.

[8] Gryboś, P. et al. (2006). DEDIX - development of fully integrated multichannel ASIC for high count rate digital X-ray imaging systems. In 2006 IEEE Nuclear Science Symposium Conference Record. IEEE, 693696.

[9] Dubecký, F. et al. (2005). Digital X-ray portable scanner based on monolithic semi-insulating GaAs detectors. Nuclear Instruments and Methods in Physics Research A , 546, 118-124. 